\title{
MULTICUSP ION SOURCE FOR INDUCTION LINAC APPLICATIONS
}

\author{
$\underline{\text { J. Reijonen }}{ }^{\dagger}$, M. Eardley, R. Keller, J. Kwan, K. N. Leung, D. Pickard", R. Thomae \\ and M. D. Williams, LBNL, University of California, Berkeley, CA 94720, USA
}

\begin{abstract}
At LBNL we are investigating the use of gaseous ion sources for induction linac applications such as heavy ion inertial fusion. Typical requirements for the ion source is to produce $20 \mu \mathrm{s}$ pulses with a rise-time of $2 \mu \mathrm{s}$. The current density should be greater than $100 \mathrm{~mA} / \mathrm{cm}^{2}$ at a duty cycle of $10 \mathrm{~Hz}$ [1]. Noble gases such as krypton, neon and xenon will be used. The source used for the measurements described in this paper was a standard 10 $\mathrm{cm}$ in diameter multicusp source with RF-discharge. Current densities of more than $400 \mathrm{~mA} / \mathrm{cm}^{2}$ at RF-power levels of $20 \mathrm{~kW}$ using neon discharge were demonstrated. With heavier elements such as $\mathrm{Ar}, \mathrm{Kr}$ and $\mathrm{Ne}$, current densities of $200-300 \mathrm{~mA} / \mathrm{cm}^{2}$ were obtained at the same extraction voltage of $20 \mathrm{kV}$. By using a starter filament, the rise-time has been reduced from $20 \mu$ s to $5 \mu$ s. Beam emittance was measured using a pepper-pot device. An argon beam showed that the normalized emittance is of the order of $0.1 \pi \mathrm{mm}$-mrad.
\end{abstract}

\section{INTRODUCTION}

For some years there have been studies to produce energy by Heavy Ion Fusion (HIF). There are proposals to accelerate heavy ions in a multi-channel induction linac. At LBNL, the Plasma and Ion Beam Technology Group has been investigating the use of a gaseous ion source for production of the heavy ion beam. RF-multicusp sources [2] have been reported to deliver high current density of positive and negative ions [3]. The advantage of the RFdriven plasma is the use of various different gases and metallic elements for ion production at high current density. In this paper we present measurements on inert gas elements $\mathrm{Ne}, \mathrm{Ar}, \mathrm{Kr}$ and $\mathrm{Xe}$.

\section{EXPERIMENTAL SETUP}

\subsection{Source setup}

The RF-amplifier is a pulsed $65 \mathrm{~kW}$ supply with maximum duty factor of $0.5 \%(100 \mu \mathrm{s}, 50 \mathrm{~Hz})$ at frequency of $2 \mathrm{MHz}$. The RF-power is delivered through a $50 \Omega$ coaxial transmission line to the impedance matching network. The purpose of the matching network is to match the $50 \Omega$ impedance of the amplifier to the impedance of antenna immersed to the plasma. To aid starting the

\footnotetext{
" Work is supported by a LBNL LDRD grant and by US DOE under contract No. DE-AC03-76SF00098

†Email: reijonen@mh1.lbl.gov

${ }^{\ddagger}$ Permanent address: Department of Applied Physics, Stanford University
}

plasma, a negatively biased tungsten filament, with respect to the source body is used.

All experimental measurements were carried out using a standard $10 \mathrm{~cm}$ multicusp source, shown in Fig 1. The plasma chamber has an inner diameter of $10 \mathrm{~cm}$ and is surrounded by $20 \mathrm{SmCo}$ magnet columns. Two pairs of magnets in the source back plate enhance the cusp confinement. Plasma is generated by a two-turn quartzantenna. The gas is introduced to the plasma chamber through a needle valve and the absolute pressure in the source is measured by a capacitance manometer. A small starter filament was used to feed seed electrons to the source.

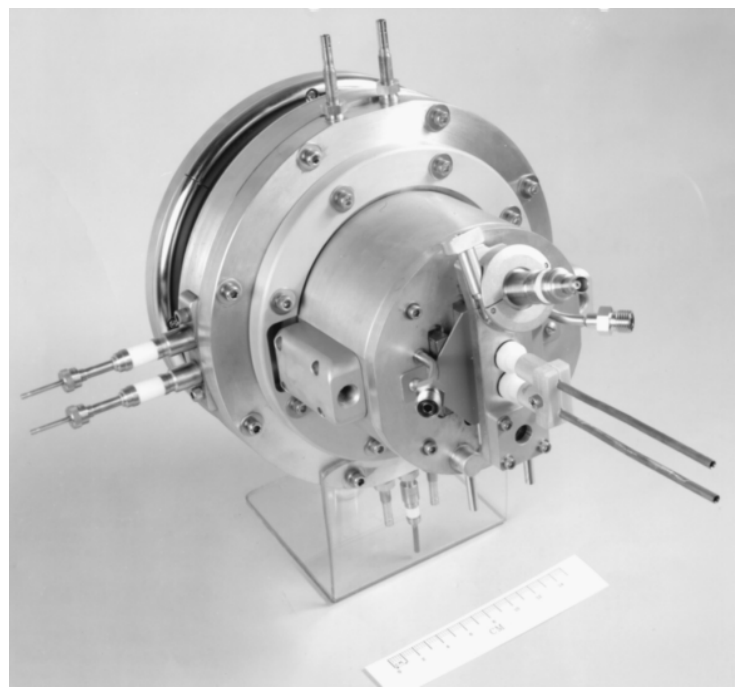

Figure 1. RF driven $10 \mathrm{~cm}$ in diameter multicusp ion source with starter filament.

The extraction system consists of a plasma electrode with an aperture of $3 \mathrm{~mm}$ for current density measurements and an aperture of $5.5 \mathrm{~mm}$ for emittance measurements. This is to simulate the proposed hole size of the HIF source. The faraday cup was located $5 \mathrm{~cm}$ from the grounded second electrode. The faraday cup is magnetically shielded to suppress the secondary electrons.

The beam emittance was determined using a "pepper-pot" measurement device. This device consists of a discriminator and a film holder plate. The discriminator is a thin plate with small holes drilled to form a certain pattern. Tantalum was used as a discriminator material. 
The hole diameter and the thickness of the plate was 200 $\mu \mathrm{m}$. The film is placed at a distance of $2 \mathrm{~cm}$ from the discriminator. Different film materials like kapton, tantalum, stainless steel, mylar and aluminium coated mylar were used. It was found that for our beam parameters a mylar film was the most advantageous.

\section{EXPERIMENTAL RESULTS}

\subsection{Current Density Measurement}

The ion current was measured using four different gases: $\mathrm{Ne}, \mathrm{Ar}, \mathrm{Kr}$ and $\mathrm{Xe}$. The current was measured as a function of acceleration voltage. In Fig 2 the results for the krypton beam is shown. From this result the saturation current is determined.

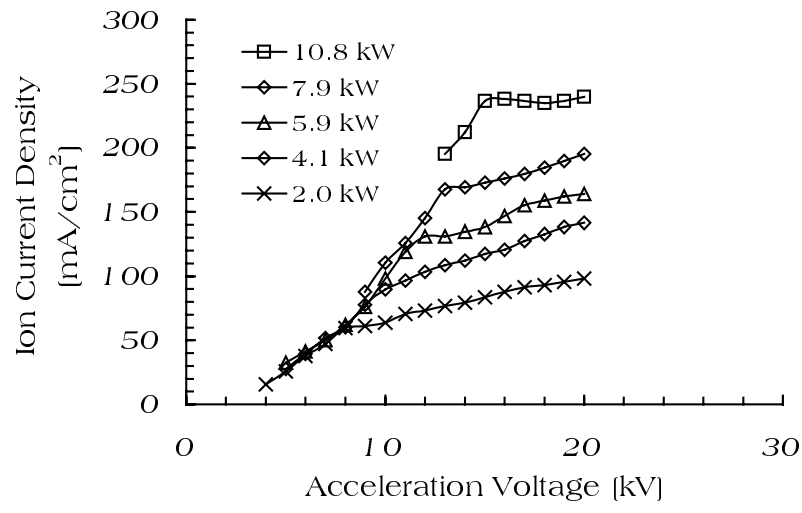

Figure 2: Extracted krypton beam current as a function of acceleration gap voltage. The saturation current is determined from this graph.

In Fig. 3 the current density as a function of RF-power for the four elements is shown. The pressure is selected so that it gives the maximum ion current. The current density is calculated from the measured saturation current values. In these measurements, it is found that the neon gas required higher pressure to sustain stable plasma.

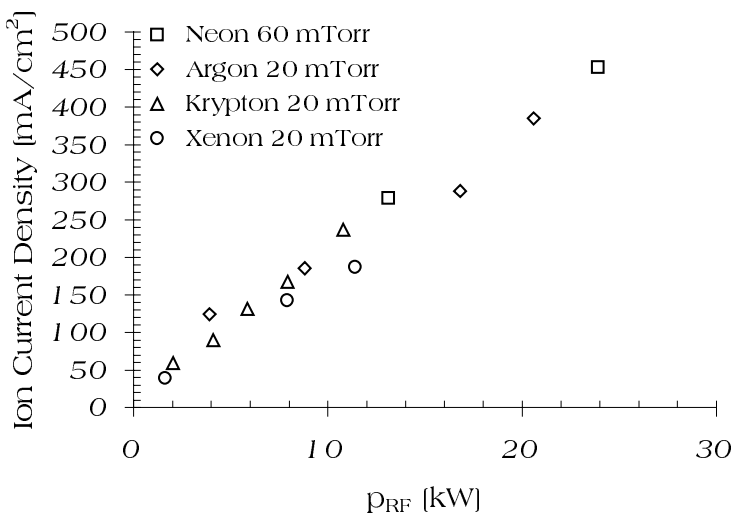

Figure 3: Current density as a function of RF-power for different elements.

\subsection{Rise-time measurement}

The rise-time of the pulses is measured using $80 \mu \mathrm{s}$ discharge pulse. Two gases were used, namely Neon and Krypton. The measured rise-times are displayed in Fig 4. For the rise-time measurement the starter filament position was optimized to achieve stable plasma at the low source pressure.

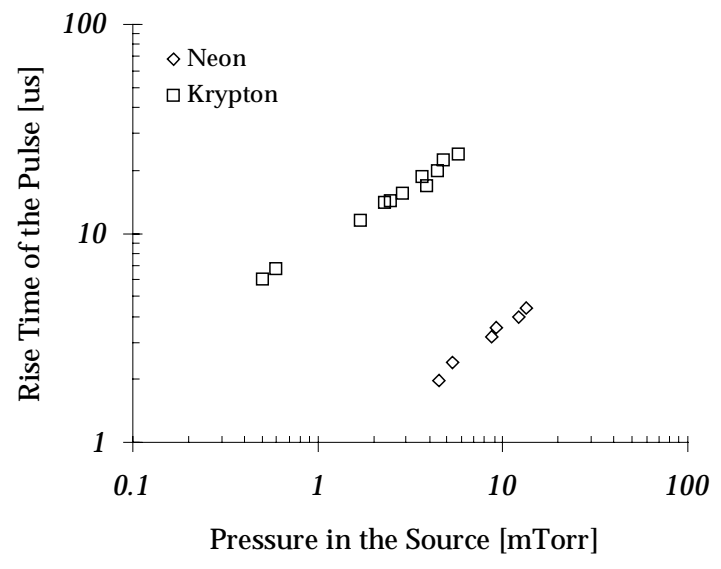

Figure 4: Rise-time of discharge pulses with two different gases as a function of source pressure. In the case of neon gas the rise-time is order of magnitude smaller compared to krypton. The shorter rise-time may be due to the higher mobility of neon ions.

\subsection{Emittance measurement}

The beam emittance was measured using a "pepper-pot" device. This device consists of a discriminator, which is a thin plate where tiny holes are drilled. Behind the plate is a film, where beamlets form a pattern. From the film patterns the emittance can be determined. For this purpose the patterns were measured by means of a microscope. An ellipse was fitted around the measured data points. The equation [4] for an ellipse is

$$
r^{\prime}= \pm \frac{B}{A} \sqrt{A^{2}-r^{2}}+C r
$$

Where A, B and C are variables that must be fitted to the measured points in the r-r' phase space. The $r$ and r' are the radius of the beamlet from the beam axis and the angle of the beamlet respectively. The emittance in the r-r' plane is then calculated using A and B simply

$$
\varepsilon=\pi A \cdot B
$$

In Fig 5 an example of such a fit is shown for an argon beam. 


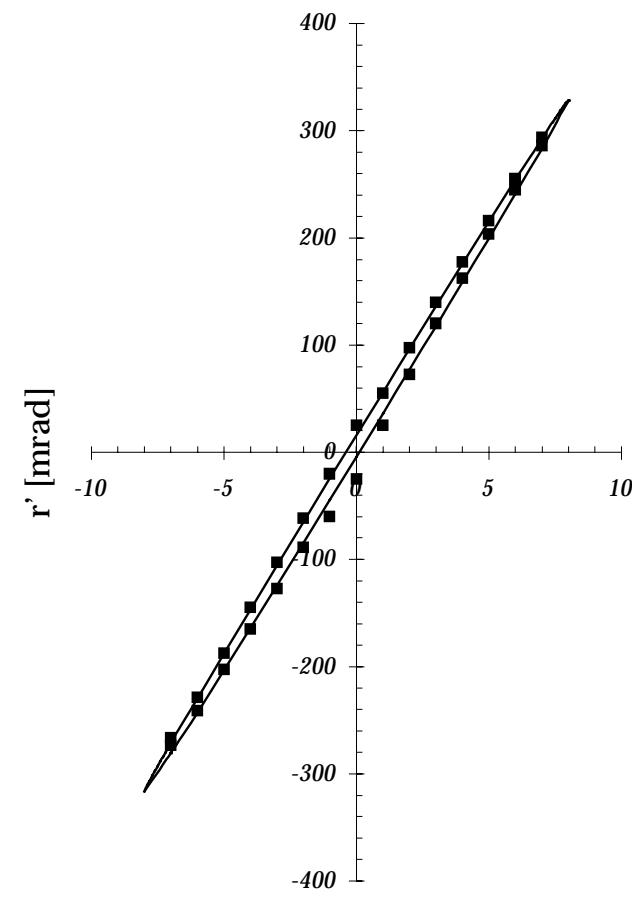

$\mathrm{r}[\mathrm{mm}]$ between neon and krypton can be explained because of the higher mobility of the neon as a lighter element.

The emittance of the pulsed $(0.2 \%$ duty factor $) 20 \mathrm{kV}$ argon beam was measured to be $105 \pi-\mathrm{mm}$-mrad, which corresponds to a normalised emittance of $0.1 \pi \mathrm{mm}$-mrad. The krypton beam emittance ( $80 \pi \mathrm{mm}$-mrad) is slightly smaller.

\section{REFERENCES}

[1] J. W. Kwan, "Ion Sources for Heavy Ion Fusion Induction Linacs", $12^{\text {th }}$ Inter. Symp. on Heavy Ion Inertial Fusion, Heidelberg, Germany, Sept. 1997

[2] K. N. Leung et. al., Rev. Sci. Instrum. 69 (2), 962 (1998)

[3] K. N. Leung et. al. NIM B74, 291-294 (1993)

[4] R. Keller et. al. IEEE Trans. Nucl. Sci NS-32, p. 2579 (1985)

Figure 5. The emittance plot of a $20 \mathrm{kV}$ argon beam at $0.2 \%$ duty factor.

The normalised emittance for $20 \mathrm{kV}$ argon beam is of the order of $0.1 \pi-\mathrm{mm}$-mrad and for $20 \mathrm{kV}$ krypton beam, $0.08 \pi$-mm-mrad.

\section{DISCUSSION}

We have demonstrated that it is possible to generate current density of $100 \mathrm{~mA} / \mathrm{cm}^{2}$ at a RF-power level of 5 $\mathrm{kW}$ for all gases. The current density increases linearly in the measured power range, i.e. even higher current densities can be achieved using higher RF-power. Furthermore in the multicusp ion source, the plasma is known to be uniform in large area in the extraction region, which allows one to use a multiaperture extraction system for high current applications like HIF.

For the current density measurements presented, the pressure in the source was fairly high (order of few tens of mTorr). This can be reduced significantly by optimising the starter filament position and current. The higher pressure, in the case of neon (compared to krypton, argon and xenon) is due to the low ionisation cross-section of neon

In the rise-time measurements, the chemically etched tip of the filament was positioned behind the RF-antenna coil in cusp field free region. This enables the operation of the source at lower pressure. The difference in the rise-times 